\title{
Channel Estimation for SCM-OFDM Systems by Modified Kalman Filter
}

\author{
Tao Peng ${ }^{1}$, Yue Xiao ${ }^{1}$, Shaoqian $\mathrm{Li}^{1}$, Huaqiang Shu ${ }^{2}$, Eric Pierre Simon ${ }^{2}$ \\ ${ }^{1}$ Nation Key Lab of Sci. and Techno. On Commun., University of Electronic Science and Technology of China, Chengdu, China \\ ${ }^{2}$ IEMN Lab, TELICE Group University of Lille, Lille, France \\ Email: tpeng.cn@gmail.com
}

Received July 2013

\begin{abstract}
In this paper, the problem of channel estimation for superposition coded modulation-orthogonal frequency division multiplexing (SCM-OFDM) systems over frequency selective channels is investigated. Assuming that the path delays are known, a new channel estimator based on modified Kalman filter algorithms is introduced for the estimation of the multipath Rayleigh channel complex gains (CG). In the simulation, the mean square error (MSE) and bit-error-rate (BER) performances are given to verify the effectiveness of the Kalman estimation algorithms for SCM-OFDM systems.
\end{abstract}

Keywords: Superposition Coded Modulation (SCM); Orthogonal Frequency Division Multiplexing (OFDM); Channel Estimation; Kalman Filter

\section{Introduction}

As a kind of non-orthogonal multiple access scheme, interleave division multiple access (IDMA) was developed by Ping et al. [1,2], in which random interleavers were employed as the only means of user separation. In general, IDMA outperforms conventional code division multiple access (CDMA) in terms of power and bandwidth efficiency. The key innovation of IDMA is the introduction of low-rate channel coding, chip-level interleaving and low-complexity multiuser detection.

Motivated by the concept of IDMA, superposition coded modulation (SCM) partitions the data to multi layer, where each layer is treated by a user equivalently. The low-rate encoder for all layers is typically identical, and the interleaver of every layer is distinct, which is used to combat the inter-layer interference. SCM has several advantages over conventional coded modulation schemes such as trellis-coded modulation (TCM) and bit-interleaved coded-modulation with iterative decoding (BICMID). First, the transmitted signal of SCM can be approximated as a Gaussian variable according to the central

\footnotetext{
"This work was supported by the Foundation Project of National Key Laboratory of Science and Technology on Communications under Grant 9140C020404120C0201, National High-Tech R\&D Program of China ("863” Project under Grant number 2011AA01A105), National Grand Special Science and Technology Project of China under Grant No. 2010ZX03006-002-02, and the Fundamental Research Funds for the Central Universities.
}

limit theorem. Second, for adaptive modulation, the rate adaptation can be simply realized in SCM by adjusting the number of layers. Furthermore, a low-cost chip-bychip iterative detection algorithm can be adopted in SCM, where the complexity is independent of the number of layers $[3,4]$. Furthermore, SCM can be combined with orthogonal frequency division multiplexing (OFDM) to combat the frequency selective fading and improve the throughput [5].

For signal detection in the receiver, reliable channel information is needed. There have only been few literatures regarding channel estimation for IDMA and SCM systems. For example, least square (LS) and minimum mean square error (MMSE) algorithms are employed to estimate channel response of IDMA systems [6,7]. However, these estimation algorithms perform per-user channel estimation using pilot symbols in the frequency domain, which lead to poor estimation performance.

To alleviate this problem, channel estimation for SCMOFDM systems is investigated in this paper. The estimation of physical channel parameters includes estimating multipath delays and multipath complex gains (CGs). It is well known that the path delays are quasi-invariant over several OFDM blocks whereas the CGs may change significantly even within one OFDM block. Therefore, the delays are assumed to be perfectly estimated and only the problem of CGs estimation is considered in this paper. Due to the excellent estimation performance of Kalman 
filter [8], a new channel estimator based on modified Kalman filter algorithms is obtained to estimate the multipath Rayleigh channel CGs. In the final, simulation results verify that the Kalman estimation algorithms can greatly improve the mean square error (MSE) performance compared to the LS and MMSE algorithms, and can achieve almost the same bit-error-rate (BER) performance as the ideal channel estimation.

The rest of this paper is organized as follows. Section II introduces the system model of SCM-OFDM systems adopted in this paper. In Section III, channel model and Kalman channel estimation algorithms are presented for SCM-OFDM systems. Simulation results are provided in Section IV to demonstrate the effectiveness of the estimation algorithms. The conclusions are drawn in Section V.

\section{System Model}

\subsection{Transmitter of a SCM-OFDM System}

The superposition coded modulation scheme with $K$ layers is shown in Figure 1. A binary data sequence $d$ is first serial-to-parallel converted into $K$ subsequences. Then the data of each layer is encoded, interleaved and modulated independently. Finally, all the data of $K$ layers are linearly superimposed to transmission. For layer- $k$, the data sequence $d_{k}$ is first encoded by a low-rate encoder, resulting in a coded sequence $c_{k}$ of length $N$. Then the coded sequence $c_{k}$ is interleaved by a distinct chip-level interleaver $\pi_{k}$ to produce a permutated sequence $\bar{c}_{k}$. After interleaving, the randomly sequence $\boldsymbol{C}_{k}$ is modulated to $X_{k}$ by binary phase shift keying (BPSK). The output signal is a linear superposition of $K$ independently coded symbols

$$
X(m)=\sum_{k=1}^{K} X_{k}(m), \quad 0 \leq m \leq N-1 .
$$

Then the superposition signal is fed into an inverse fast Fourier transform (IFFT) modulator. After IFFT and cyclic prefix (CP) insertion, the time domain transmitted signal can be represented as

$$
x(n)=\frac{1}{N} \sum_{m=0}^{N-1} X(m) e^{j 2 \pi m n / N}, \quad-N_{g} \leq n \leq N-1
$$

where $N$ is the total number of subcarriers and $N_{g}$ is the length of CP.

\subsection{Receiver of a SCM-OFDM System}

Since each layer of SCM systems can be treated as one user of multi-user systems, the suboptimal iterative detection [2] can be applied in SCM systems. The receiver of SCM-OFDM is composed by a multi-layer detector (MLD) and $K$ decoders (DECs), which are applied to solve inter-layer interference and the coding constraint separately. The receiver performs the iterative processes to update the extrinsic information between ESE and DECs. After the last iteration, the DECs produce hard decisions towards information bits. The receiver of SCMOFDM is illustrated in Figure 2.

The transmitted signals will pass through the multipath fading channels and be corrupted by additive white Gaussian noise (AWGN). At the receiver, after FFT processing and $\mathrm{CP}$ removal, the frequency domain received signal is given by

$$
\begin{aligned}
R(m) & =X(m) H(m)+W(m) \\
& =H(m) \sum_{k=1}^{K} X_{k}(m)+W(m),
\end{aligned}
$$

where $H(m)$ is the channel frequency response at the $m$ th subcarrier, and $W(m)$ is the AWGN with zero mean and variance $\sigma^{2}$.

To detect the $m$ th chip in the $k$ th layer, the received signal can be rewritten as

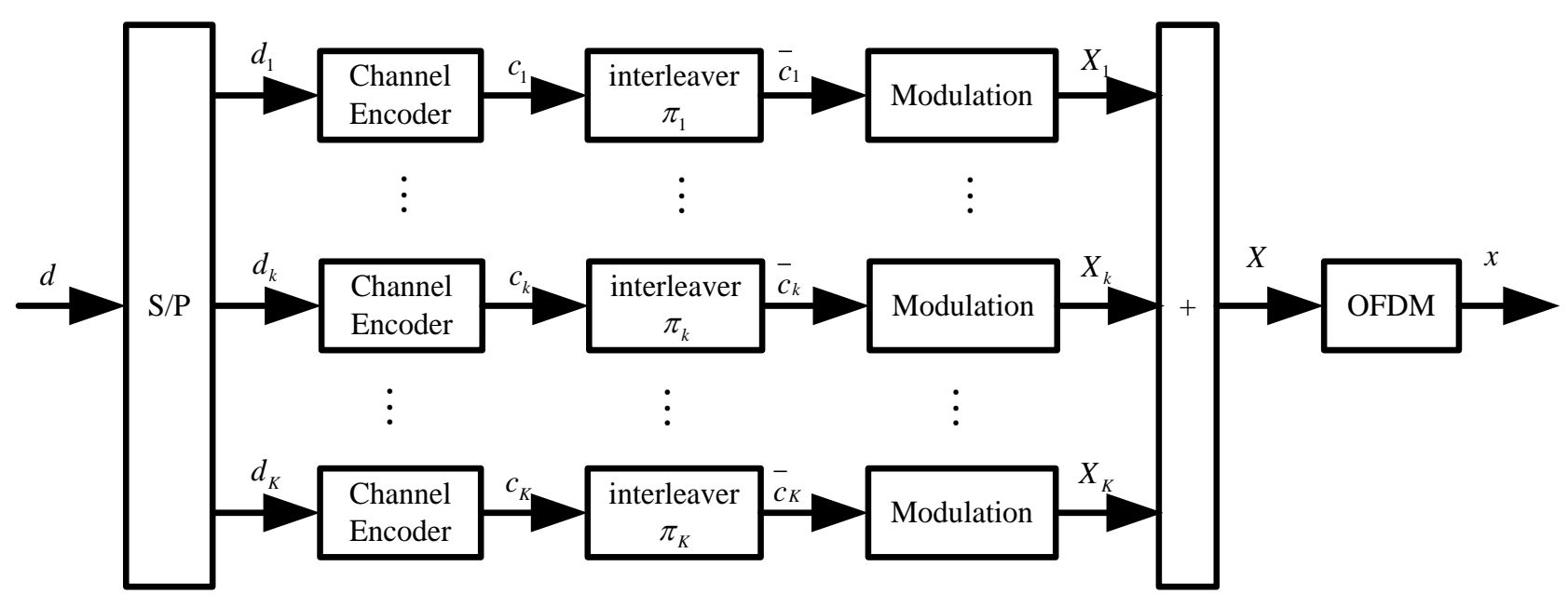

Figure 1. Transmitter structure of a SCM system. 


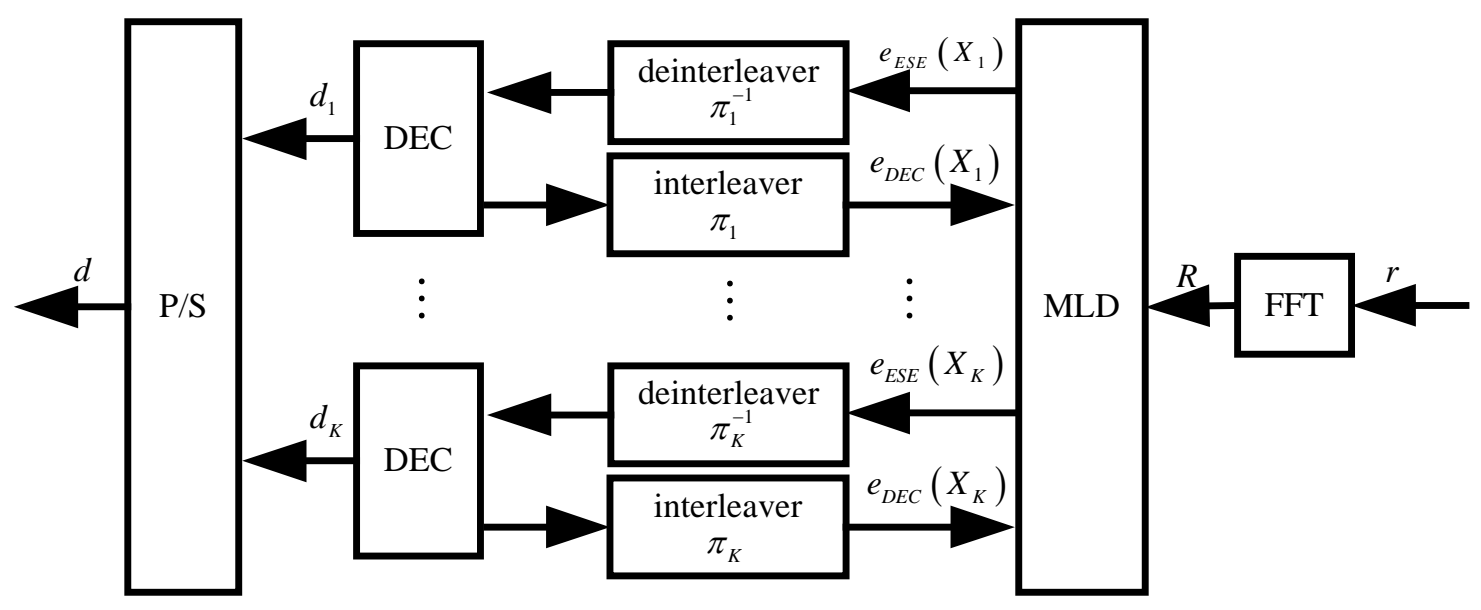

Figure 2. Receiver structure of a SCM system.

$$
\begin{aligned}
R(m) & =H(m) X_{k}(m)+\sum_{k^{\prime}=1, k^{\prime} \neq k}^{K} H(m) X_{k^{\prime}}(m)+W(m) \\
& =H(m) X_{k}(m)+\zeta_{k}^{M L I}(m)
\end{aligned}
$$

where $\zeta_{k}^{M L I}(m)$ is the total interference term with respect to layer- $k$ on subcarrier- $m$, consisted of both inter-layer interference and noise.

According to the central limit theorem, $\zeta_{k}^{M L I}(m)$ is approximated as a Gaussian random variable with mean $E\left(\zeta_{k}^{M L I}(m)\right)$ and variance $\operatorname{Var}\left(\zeta_{k}^{M L I}(m)\right)$. Based on the definition of extrinsic LLR, the output of ESE detector is calculated by

$$
e_{E S E}\left(X_{k}(m)\right)=2 H(m) \frac{R(m)-E\left(\zeta_{k}^{M L I}(m)\right)}{\operatorname{Var}\left(\zeta_{k}^{M L I}(m)\right)}
$$

where $E\left(\zeta_{k}^{M L I}(m)\right)$ and $\operatorname{Var}\left(\zeta_{k}^{M L I}(m)\right)$ can be obtained by the mean and variance of $X_{k}(m)$.

At each iteration, by the priori LLRs feedback from the DECs, the mean and variance of $X_{k}(m)$ can be calculated as follows

$$
\begin{gathered}
E\left(X_{k}(m)\right)=\tanh \left(e_{D E C}\left(X_{k}(m)\right) / 2\right), \\
\operatorname{Var}\left(X_{k}(m)\right)=1-\left(E\left(X_{k}(m)\right)\right)^{2} .
\end{gathered}
$$

In the above detection algorithm, the channel information will be utilized at every iterative step. And the system performance can be enhanced by improving the accuracy of the channel estimation. Therefore, channel parameter estimation is an important task for SCM-OFDM systems.

\section{Channel Estimation Based on Modified Kalman Filter}

\subsection{Channel Model}

The Rayleigh fading channel model with Jakes’s Doppler spectrum is the most accepted random model to represent temporal variations of the equivalent baseband channel CG in wireless communication. Thus, this channel model is considered in this paper with a delay spread $L$. The normalized Doppler frequency is denoted as $f_{D} T$, where $f_{D}$ represents the maximum Doppler frequency shift and $T$ is an OFDM symbol period.

After transmission over a multipath Rayleigh channel, the received OFDM symbol is given in the frequency domain (after removing CP and taking FFT) by a matrix form as

$$
\mathbf{R}=\mathbf{H X}+\mathbf{W}
$$

where $\mathbf{R}=[R(0), R(1), \ldots, R(N-1)]^{T}, \mathbf{X}$ and $\mathbf{W}$ are defined in a similar way as $\mathbf{R}$.

The $N \times N$ channel matrix $\mathbf{H}$ can be written in terms of physical channel parameters as

$$
[\mathbf{H}]_{n, n^{\prime}}=\frac{1}{N} \sum_{l=1}^{L}\left[e^{-j 2 \pi \frac{n^{\prime}}{N} \tau_{l}} \sum_{q=0}^{N-1} \alpha_{l}\left(q T_{s}\right) e^{j 2 \pi \frac{n^{\prime}-n}{N} q}\right]
$$

where $\tau_{l}$ is the lth path delay normalized by the sampling time, $T_{s}$ is the sampling time, and $\alpha_{l}$ is the lth path CGs.

When the channel is assumed to be time invariant within a block, the channel matrix $\mathbf{H}$ can be simplified to a diagonal matrix denoted as

$$
\left[\mathbf{H}_{s}\right]_{n, n}=\sum_{l=1}^{L} \alpha_{l}\left(n T_{s}\right) e^{-j 2 \pi \frac{n}{N} \tau_{l}} .
$$

Let us define the $N \times L$ matrix $\mathbf{F H}$ and the $L \times N$ matrix $\boldsymbol{\alpha}$ as follows,

$$
\begin{gathered}
{[\boldsymbol{\alpha}]_{l, \boldsymbol{*}}=\left[\alpha_{l}(0), \alpha_{l}(T), \ldots, \alpha_{l}\left((N-1) T_{s}\right)\right]} \\
{[\mathbf{F H}]_{n, l}=e^{-j 2 \pi \tau_{l} n / N}}
\end{gathered}
$$

Then substituting (11) and (12) in (10) yields

$$
\mathbf{H}_{s}=\mathbf{F H} \cdot \boldsymbol{\alpha}
$$


After obtained the path delays and CGs, the channel matrix can be calculated by (13). Note that $\boldsymbol{\alpha}$ will be time-varying actually if the normalized Doppler frequency $f_{D} T$ is larger. In this paper, $\boldsymbol{\alpha}$ is assumed to be fixed when the channel is time-invariant. And the delays are assumed to be perfectly estimated and only the problem of CGs estimation is considered.

\subsection{Modified Kalman Filter Algorithms}

In this subsection, the problem of channel estimation for SCM-OFDM systems over time invariant channels is considered. Assuming that the path delays are known, a new channel estimator based on modified Kalman filter algorithms is obtained to estimate the channel CGs. In this estimation algorithm, each CG within one OFDM symbol can be estimated by using a Kalman filter recursively. For clarity, the modified Kalman filter algorithms are summarized in the following, where two different Kalman initialization methods are given as algorithm 1 and algorithm 2.

1) Initialization:

$$
\begin{aligned}
& \mathbf{S}=\left\{\begin{array}{l}
\operatorname{besselj}\left(0,2 \pi f_{d} T\right), \quad \text { algorithm } 1 \\
\sqrt{1-4\left(\left(\pi f_{d} T\right)^{4} \sigma^{2}\right)^{1 / 3}}, \text { algorithm } 2
\end{array}\right. \\
& \mathbf{U}=\left(1-\mathbf{S}^{2}\right) \operatorname{diag}\left(\mathbf{P}_{v}\right) \\
& \mathbf{P}_{0}=\operatorname{zeros}(L, L) \\
& \mathbf{K}_{a}=\mathbf{F}_{p} \operatorname{diag}\left(N_{p}\right)
\end{aligned}
$$

2) Recursive Estimation: for symbol index $i=1,2, \ldots$, Prediction step:

$$
\begin{aligned}
& \widehat{\boldsymbol{\alpha}}_{i \mid i-1}=\widehat{\mathbf{S}}_{i-1} \\
& \mathbf{P}_{i \mid i-1}=\mathbf{P}_{i-1}+\mathbf{U} \\
& \mathbf{K}_{i}=\mathbf{P}_{i \mid i-1} \mathbf{K}_{a}^{\prime} /\left(\mathbf{K}_{a} \mathbf{P}_{i \mid i-1} \mathbf{K}_{a}^{\prime}+\sigma^{2} \operatorname{eye}\left(N_{p}\right)\right) \\
& \text { Updata step: } \\
& \hat{\boldsymbol{\alpha}}_{i}=\widehat{\boldsymbol{\alpha}}_{i \mid i-1}+\mathbf{K}_{i}\left(\mathbf{R}_{p}^{\prime}-\mathbf{K}_{a} \widehat{\boldsymbol{\alpha}}_{i \mid i-1}\right) \\
& \mathbf{P}_{i}=\mathbf{P}_{i \mid i-1}-\mathbf{K}_{i} \mathbf{K}_{a} \mathbf{P}_{i \mid i-1}
\end{aligned}
$$

In the above modified Kalman filter algorithms, the estimation for the channel CG of each OFDM symbol is performed continuously and recursively. With the increasing number of estimation, the estimation performance will become more and more accurate.

\section{Simulation Results}

In this section, computer simulations are carried out to verify the effectiveness of the Kalman estimation algorithms for SCM-OFDM systems. Simulations are performed for a SCM-OFDM system with four layers, employing BPSK modulation. System parameters are as follows, $N=128$ subcarriers, $N_{\mathrm{g}}=N / 8, N_{\mathrm{p}}=N / 8$ pilots, and $1 / T_{\mathrm{s}}=2 \mathrm{MHz}$. The channels are assumed to be time-invariant, thus the normalized Doppler frequency is selected as $f_{D} T=0.0001$. Two Rayleigh channel model [9,10] are chosen as shown in Tables 1 and 2.

Figures 3 and $\mathbf{4}$ show the MSE performances of the Kalman channel estimation algorithms compared to LS and MMSE estimation algorithms in channel model A and model B. It can be found that the performances of the Kalman algorithms are significantly better than that of the LS and MMSE algorithms. And the performance gaps between the Kalman algorithms and others become greater with increasing SNR. Moreover, the Kalman algorithm 2 can achieve more excellent estimation performance than Kalman algorithm 1 in both channel models.

Figure 5 depicts the BER performances of the Kalman channel estimation algorithms for data detection compared to LS and MMSE estimation algorithms. In this figure, the ideal estimation algorithm is included, where the exact channel information is perfectly known in the receiver. From this figure, it can be observed that the Kalman algorithms can enhance the BER performance obviously rather than the LS and MMSE algorithms. Meanwhile, the performance of the Kalman algorithm 2 is almost the same as that of the ideal algorithm.

Table 1. Multipath channel model A.

\begin{tabular}{ccc}
\hline Path number & Average (dB) & Delay (Ts) \\
\hline 0 & -7.219 & 0 \\
1 & -4.219 & 0.4 \\
2 & -6.219 & 1 \\
3 & -10.219 & 3.2 \\
4 & -12.219 & 4.6 \\
5 & -14.219 & 10 \\
\hline
\end{tabular}

Table 2. Multipath channel model B.

\begin{tabular}{ccc}
\hline Path number & Average (dB) & Delay (Ts) \\
\hline 0 & 0 & 0 \\
1 & -1 & 0.6 \\
2 & -9 & 1.4 \\
3 & -10 & 2.2 \\
4 & -15 & 3.5 \\
5 & -20 & 5 \\
\hline
\end{tabular}

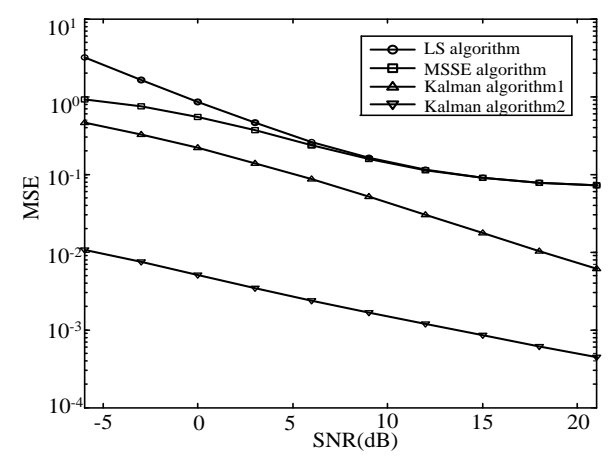

Figure 3. MSE performances of the Kalman estimation algorithms for SCM-OFDM systems in channel model A. 


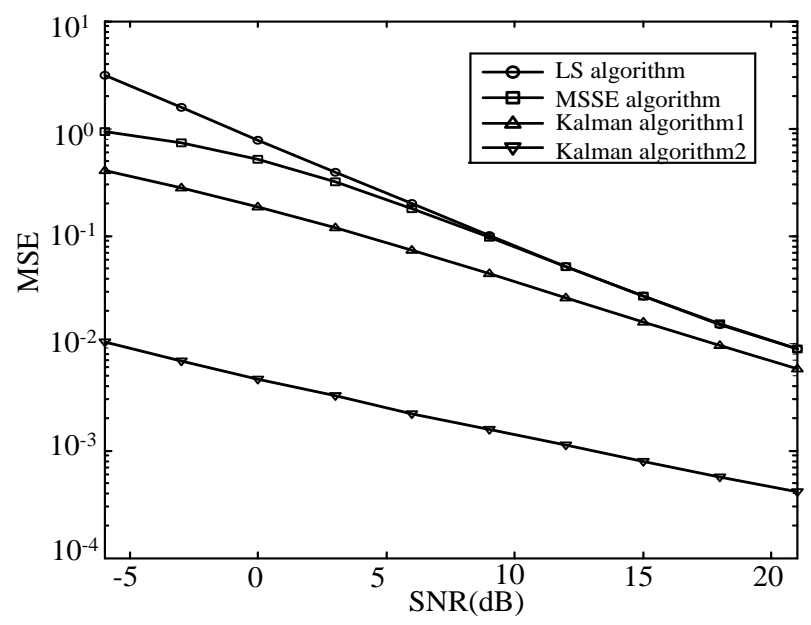

Figure 4. MSE performances of the Kalman estimation algorithms for SCM-OFDM systems in channel model B.

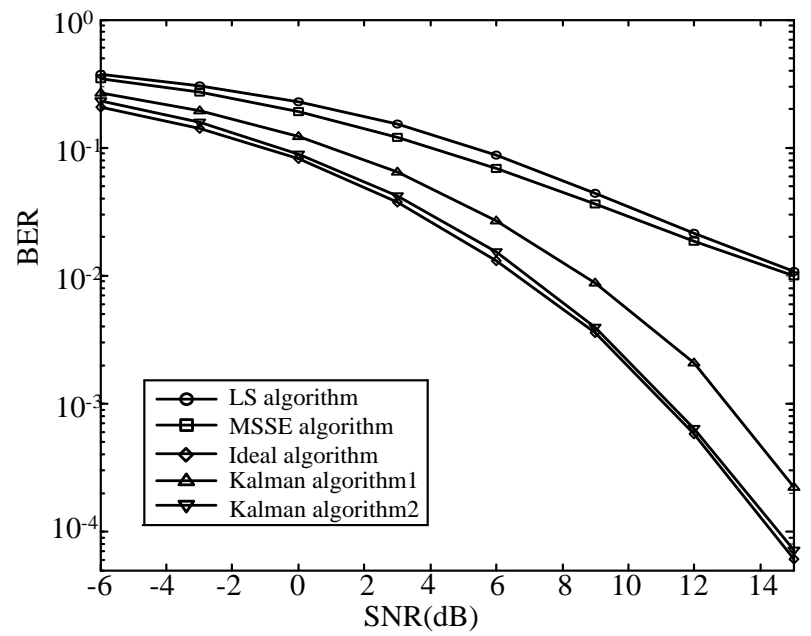

Figure 5. BER performances of the Kalman estimation algorithms for SCM-OFDM systems in channel model A.

\section{Conclusion}

In this paper, channel estimation for SCM-OFDM systems is investigated over frequency selective channels. Compared to LS and MMSE algorithms, the Kalman algorithms can significantly improve the MSE and BER performances. More specifically, the Kalman algorithm 2 can achieve the performance very close to the ideal algorithm.

\section{REFERENCES}

[1] P. Li, L. H. Liu, K. Y. Wu and W. K. Leung, "A Unified Approach to Multiuser Detection and Space-Time Coding with Low Complexity and Nearly Optimal Performance,” Proceedings of 40th Allerton Conference, 2002, pp. 170179.

[2] P. Li, L. H. Liu, K. Y. Wu and W. K. Leung, "Interleave division multiple access," IEEE Transaction on Wireless Communication, Vol. 5, No. 4, 2006, pp. 938-947.

[3] J. Tong and P. Li, "Iterative Decoding of Superposition Coding," Proceedings of 4th International Symposium on Turbo Codes, 2006.

[4] J. Tong, P. Li and X. Ma, "Superposition Coded Modulation with Peak-Power Limitation," IEEE Transactions on Information Theory, Vol. 55, No. 6, 2009, pp. 2562-2576. http://dx.doi.org/10.1109/TIT.2009.2018224

[5] J. Tong, P. Li, Z. H. Zhang and W. K. Bhargava, "Iterative Soft Compensation for OFDM Systems with Clipping and Superposition Coded Modulation," IEEE Transactions on Communications, Vol. 58, No. 10, 2010, pp. 2861-2870.

http://dx.doi.org/10.1109/TCOMM.2010.083110.09296

[6] H. Schoeneich and P. A. Hoeher, "Iterative Pilot-Layer Aided Channel Estimation with Emphasis on Interleave-Division Multiple Access Systems," EURASIP Journal on Advances in Signal Processing, Vol. 2006, 2006, pp. 1-15. http://dx.doi.org/10.1155/ASP/2006/81729

[7] S. Suyama, H. Suzuki, K. Fukawa and L. Zhang, "Iterative Multiuser Detection with Soft Decision-Directed Channel Estimation for MC-IDMA and Performance Comparison with Chip-Interleaved MC-CDMA," IEICE IEEE Transactions on Communications, Vol. E92-B, No. 5, 2009, pp. 1495-1503.

[8] S. Ghandour-Haidar, L. Ros, J.M. Brossier, “On the Use of First-Order Autoregressive Modeling for Rayleigh Flat Fading Channel Estimation with Kalman Filter,” Signal Processing, Vol. 92, No. 2, 2012, pp. 601-606. http://dx.doi.org/10.1016/j.sigpro.2011.08.014

[9] E. P. Simon, L. Ros, H. Hijazi and M. Ghogho, "Joint Carrier Frequency Offset and Channel Estimation for OFDM Systems via the EM Algorithm in the Presence of Very High Mobility,” IEEE Transaction on Signal Process, Vol. 60, No. 2, 2012, pp. 754-765. http://dx.doi.org/10.1109/TSP.2011.2174053

[10] E. P. Simon, L. Ros, H. Hijazi, et al., "Joint Carrier Frequency Offset and Fast Time-Varying Channel Estimation for MIMO-OFDM Systems," IEEE Transactions on Vehicular Technology, Vol. 60, No. 3, 2011, pp. 955-965. http://dx.doi.org/10.1109/TVT.2011.2104970 